\title{
CINÉTICA DE REDUÇÃO DO CATALISADOR $\mathrm{CuO} / \mathrm{ZnO} / \mathrm{Al}_{2} \mathrm{O}_{3}$
}

\section{Marcio Nele*}

Escola de Química, Universidade Federal do Rio de Janeiro, Ilha do Fundão, 21949-900 Rio de Janeiro - RJ, Brasil

\section{Esteban Lopez Moreno}

Diretoria de Extensão, Fundação Centro de Ciências e Educação Superior a Distância do Estado do Rio de Janeiro,

Rua Visconde de Niterói, 1364, 20943-001 Rio de Janeiro - RJ, Brasil

Heloysa Martins Carvalho Andrade

Departamento de Química Geral e Inorgânica, Instituto de Química, Universidade Federal da Bahia, 40170-290 Salvador - BA, Brasil

Recebido em 26/7/06; aceito em 18/5/07; publicado na web em 16/10/07

\begin{abstract}
REDUCTION KINETICS OF A CuO/ZnO/Al $\mathrm{O}_{3}$ CATALYST. The reduction kinetics of a $\mathrm{CuO} / \mathrm{ZnO} / \mathrm{Al}_{2} \mathrm{O}_{3}$ catalyst by hydrogen was investigated isothermally and by temperature programmed reduction (TPR). Two reducible $\mathrm{Cu}^{2+}$ species were detected; the first one was identified as $\mathrm{CuO}$ bulk and the other as $\mathrm{Cu}^{2+}$ strongly interacting with alumina, possibly in the form of copper aluminate. The activation energies for the reduction of these two species were 60 and $90 \mathrm{~kJ} \mathrm{~mol}^{-1}$, respectively, and the reaction order with respect to hydrogen was one. The isothermal reduction data showed that the isotropic growth model is the most appropriate to describe the reaction rate data for both $\mathrm{Cu}^{2+}$ species.
\end{abstract}

Keywords: kinetics of reduction; thermoprogrammed reduction; activation energy.

\section{INTRODUÇÃO}

Catalisadores do tipo $\mathrm{CuO} / \mathrm{ZnO} / \mathrm{M}_{2} \mathrm{O}_{3}(\mathrm{M}=\mathrm{Al}, \mathrm{Cr})$ são usados em importantes processos industriais, tais como síntese de metanol, produção de hidrogênio e remoção de compostos orgânicos ${ }^{1,2}$. Este sistema catalítico tem atraído grande interesse acadêmico na elucidação da sua preparação, em especial da etapa de co-precipitação na qual importantes mudanças físico-químicas ocorrem, tais como a formação de diferentes fases de carbonatos, hidróxidos e hidroxicarbonatos, a depender das condições de preparação ${ }^{1,3,4}$. O processo de preparação do catalisador $\mathrm{CuO} / \mathrm{ZnO} / \mathrm{M}_{2} \mathrm{O}_{3}(\mathrm{M}=\mathrm{Al}$, $\mathrm{Cr}$ ) é representado na Figura 1 através de um diagrama de causa e efeito, no qual as principais etapas da preparação juntamente com as suas variáveis são apresentadas.

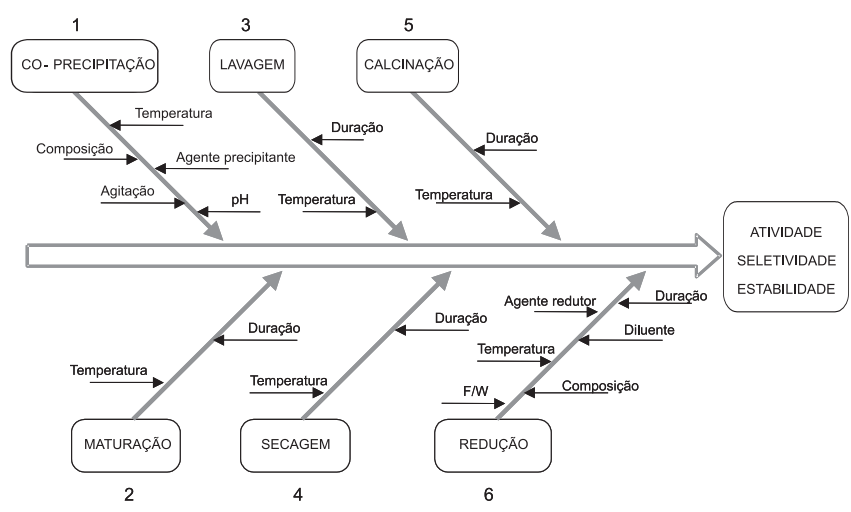

Figura 1. Diagrama de causa e efeito do processo de preparação do catalisador $\mathrm{CuO} / \mathrm{ZnO} / \mathrm{Al}_{2} \mathrm{O}_{3}$

A primeira etapa da preparação é a coprecipitação (i.e., precipitação simultânea das espécies), trata-se de um fenômeno complexo que tem forte influência sobre as propriedades finais do catalisador.

*e-mail: nele@eq.ufrj.br
A precipitação inicia-se pela nucleação, na qual íons se combinam em solução formando os cristalitos, que darão origem às partículas do precipitado. Após esta fase, dá-se o crescimento dos cristais e a precipitação propriamente dita. Durante a precipitação o meio deve ser mantido homogêneo e a temperatura deve ser cuidadosamente controlada, especialmente em misturas viscosas.

O objetivo da co-precipitação é produzir um precipitado floculado multicomponente no qual o microcristal contendo a espécie ativa é envolvido pelos cristais do precursor do suporte, com um pequeno tamanho de cristal para a espécie ativa e o suporte ${ }^{5}$. A preparação do catalisador $\mathrm{CuO} / \mathrm{ZnO} / \mathrm{Al}_{2} \mathrm{O}_{3}$ resulta em um alto grau de interdispersão dos seus componentes ${ }^{6}$.

Finalizada a precipitação, a próxima etapa é a maturação. $\mathrm{O}$ objetivo desta etapa é aguardar que as reações físico-químicas que estão ocorrendo no reator atinjam o equilíbrio, para permitir a formação de outras fases desejáveis e para promover o crescimento dos cristais. As principais variáveis deste processo são tempo e temperatura de maturação. Períodos muito longos de maturação podem determinar o crescimento excessivo dos cristais, diminuindo a área metálica ${ }^{7}$.

Após a maturação, o precipitado passa por uma etapa de lavagem para remoção dos íons indesejáveis. A lavagem merece uma atenção especial quando o agente precipitante contém sódio (ex.: carbonato de sódio). O teor residual de sódio deve ser mantido abaixo de $0,05 \%$ para que seu efeito na atividade do catalisador seja desprezível ${ }^{8}$.

Completada a lavagem, o precipitado é seco e calcinado. Nesta última etapa, a calcinação, os precursores hidróxidos e carbonatos são predominantemente transformados em óxidos, cujas características físico-químicas são fortemente dependentes das condições de aquecimento e da natureza dos precursores ${ }^{8}$.

Finalmente, o precursor passa por um tratamento de redução para que o catalisador tome a sua forma ativa, por isso essa etapa é também denominada de ativação. Durante este processo são estruturadas características importantes do catalisador, como quantidade e dispersão de espécies ativas sobre o suporte. A execução 
incorreta da redução pode levar a um desempenho inferior e uma menor vida útil do catalisador, essencialmente devido à sinterização do cobre metálico formado. A despeito da importância da etapa de redução, poucos artigos exploram a cinética de redução de sistemas catalíticos do tipo $\mathrm{CuO} / \mathrm{ZnO} / \mathrm{M}_{2} \mathrm{O}_{3}$.

$\mathrm{Naidu}^{9}$ estudou, isotermicamente em aparato termogravimétrico, a cinética de redução de um catalisador comercial $\left(\mathrm{CuO} / \mathrm{ZnO} / \mathrm{Fe}_{2} \mathrm{O}_{3}\right)$. A equação de Prout-Tompkins foi a que melhor descreveu a cinética de redução e uma energia de ativação de $49 \mathrm{~kJ} \mathrm{~mol}^{-1}$ foi encontrada para a redução por $\mathrm{H}_{2}$.

Sistemas modelo $\mathrm{CuO}, \mathrm{CuO} / \mathrm{ZnO}$ e $\mathrm{CuO} / \mathrm{Al}_{2} \mathrm{O}_{3}$ foram utilizados para estudar a cinética de redução do catalisador $\mathrm{CuO} / \mathrm{ZnO} /$ $\mathrm{Al}_{2} \mathrm{O}_{3}$. Naumov et al. ${ }^{10}$ obtiveram curvas sigmóides, atribuídas a processos autocatalíticos, para a redução isotérmica com ordem de reação unitária para a pressão parcial de $\mathrm{H}_{2}$. Concluíram que o óxido de zinco e a alumina retardam a redução do óxido de cobre, no entanto, o óxido de zinco acelera a última etapa da redução. Contrariamente a Ruggeri et al. ${ }^{11}$ que afirmaram que a alumina aumenta a reatividade do $\mathrm{CuO}$ frente à redução pelo aumento da dispersão do óxido de cobre e que, apesar disto, o óxido de zinco retarda a redução.

Bueno et al. ${ }^{12}$ estudaram a redução de catalisadores $\mathrm{CuO} / \mathrm{ZnO} /$ $\mathrm{Al}_{2} \mathrm{O}_{3}$ e $\mathrm{CuO} / \mathrm{ZnO} / \mathrm{Cr}_{2} \mathrm{O}_{3}$. Nestes sistemas o processo de redução apresentou uma energia de ativação de até $107 \mathrm{~kJ} \mathrm{~mol}^{-1}$, maior que a normalmente encontrada para o $\mathrm{CuO}$, sugerindo a formação de espécies $\mathrm{Cu}^{2+}$ estáveis. Fierro et al. ${ }^{13}$ estudaram a redução de sistemas modelo $\mathrm{CuO} / \mathrm{ZnO}$ em uma ampla faixa de composição; concluíram que o óxido de zinco promove a redução do óxido de cobre, frente ao comportamento do óxido de cobre puro.

Em um estudo anterior foram exploradas as variáveis operacionais que otimizam a análise de redução termoprogramada, utilizando como estudo de caso a redução termopragramada do sistema $\mathrm{CuO} / \mathrm{ZnO} / \mathrm{Al}_{2} \mathrm{O}_{3}{ }^{14}$. Este trabalho tem por objetivo investigar a cinética de redução do catalisador $\mathrm{CuO} / \mathrm{ZnO} / \mathrm{Al}_{2} \mathrm{O}_{3}$ por redução termoprogramada e isotermicamente, de modo a obter uma expressão para a cinética de redução do catalisador. Esta expressão permitirá avaliar a taxa de redução a uma determinada condição experimental e evitar que condições capazes de promover a sinterização do catalisador sejam utilizadas.

\section{Fundamentos teóricos: cinética de reações sólido-gás}

Uma descrição simplificada da cinética química de um sistema é baseada em uma equação definindo a relação entre a taxa de conversão $\alpha$ e o tempo ${ }^{15}$.

$\frac{d \alpha}{d t}=k_{(T, \mathbf{b})} f_{(\alpha, \mathbf{d})}$

O termo correspondente ao modelo de Arrhenius $k_{(\mathrm{T}, \mathrm{b})}$ é dependente apenas da temperatura e os parâmetros do modelo (b) são características termodinâmicas do sistema investigado. O termo cinético $f_{(\alpha, \mathbf{d})}$ depende somente do grau de conversão $(\alpha)$. Os parâmetros do modelo (d) são determinados pelo modelo cinético escolhido para descrever a reação.

Várias são as equações cinéticas que podem descrever uma curva $\alpha$ versus $t$ tipo sigmoidal, amplamente encontrada na literatura em reações sólido-gás ${ }^{16}$ sendo que dois dos modelos relevantes estão apresentados na Tabela $1^{17}$.

A equação de Avrami foi derivada admitindo núcleos randomicamente distribuídos, criados a uma taxa constante durante a reação e cujas interfaces crescem a uma taxa constante. $\mathrm{O}$ parâmetro $n$ é determinado pela forma do núcleo, ou seja pela dire-
Tabela 1. Equações capazes de descrever uma curva $\alpha$-t tipo sigmoidal

\begin{tabular}{lcc}
\hline Equação & $g_{(\alpha, \mathbf{d})}=k_{(\mathrm{T}, \mathbf{b})} \mathrm{t}$ & $f_{(\alpha, \mathbf{d})}=1 / k_{(\mathrm{T}, \mathbf{b})}(\mathrm{d} \alpha / \mathrm{dt})$ \\
\hline Avrami & {$[-\ln (1-\alpha)]^{1 / \mathrm{n}}$} & $4(1-\alpha)(-\ln (1-\alpha))^{3 / \mathrm{n}}$ \\
Prout-Tompkins & $\ln [\alpha /(1-\alpha)]+\mathrm{C}$ & $\alpha(1-\alpha)$ \\
\hline
\end{tabular}

ção preferencial de crescimento. Quando $n=2$ o núcleo tem crescimento unidimensional (aculeiforme), quando $n=3$ o núcleo cresce bidimensionalmente (núcleo planiforme) e quando $n=4$ o núcleo cresce nas três dimensões (núcleo esferiforme) ${ }^{17}$.

A equação de Prout-Tompkins foi derivada para reações de decomposição de sólidos de primeira ordem e autocatalíticas que ocorrem via formação e crescimento de núcleos na superfície e seio do material. As principais hipóteses envolvidas no desenvolvimento do modelo são: todos os núcleos estão presentes no início da reação e o crescimento dos núcleos é bidimensional ${ }^{17}$. Bond ${ }^{18} \mathrm{e}$ Voge ${ }^{19}$ utilizaram com sucesso esta equação para descrever a redução do óxido de cobre.

Outras equações baseadas em modelos geométricos ou de processo controlado pelo crescimento da interface ${ }^{20}$ podem ser utilizadas para descrever a cinética de redução. Neste tipo de modelo, assume-se que a nucleação ocorre rapidamente e o processo é governado pelo crescimento da interface. Algumas relações para casos particulares de crescimento reativo interfacial ("reactive interfacial growth model" $)^{21}$ são apresentadas a seguir:

crescimento isotrópico do cristal (modelo cúbico ou esférico):

$\frac{k_{i} t}{a_{o}}=1-(1-\alpha)^{1 / 3}$

crescimento unidimensional (modelo cilíndrico ou do paralelepípedo):

$\frac{k_{i} t}{a_{o}}=1-(1-\alpha)^{1 / 2}$

crescimento bidimensional (quando as dimensões laterais são muito maiores que a espessura do cristal):

$\frac{k_{i} t}{a_{o}}=\alpha$

onde $k_{i}$ é a constante da taxa de crescimento interfacial, a é a menor dimensão do cristal e $t$ é o tempo.

Estas equações podem descrever satisfatoriamente a redução do óxido de cobre em termos fenomenológicos, ou seja, permitem induzir sobre a forma e a velocidade de crescimento do cristal durante a redução se um dado modelo puder ser escolhido univocamente. Neste trabalho, foi escolhido o modelo que melhor descreve os nossos dados experimentais a partir da melhor equação obtida por regressão linear (critério dos mínimos quadrados).

A análise cinética foi feita, inicialmente, pelo método da constante de taxa empírica $\left(k_{e}\right)^{21}$ que é o maior coeficiente angular da curva de redução. Sabe-se que a constante de taxa empírica $\left(k_{e}\right)$ é uma complexa função da constante da taxa de germinação $\left(k_{g}\right)$ e da constante da taxa de crescimento interfacial $\left(k_{i}\right)$, além da pressão (concentração de agente redutor) e temperatura do sistema (Equação 5). Esta análise inicial permite apenas analisar os fatores que levam a uma maior ou menor constante da taxa, sem nenhuma inferência possível em relação aos mecanismos de crescimento do cristal. 
$k_{e}=f\left(P, T, k_{i}, k_{g}\right)$

\section{PARTE EXPERIMENTAL}

Os experimentos para a determinação da cinética de redução foram realizados em um aparato experimental padrão para a execução de experimentos de redução termoprogramada ${ }^{22}$. Basicamente este equipamento permite a coleta de dados de consumo de hidrogênio durante a redução isotérmica ou termoprogramada. Dois procedimentos foram utilizados para o estudo isotérmico: procedimento 1: a amostra é aquecida em $\mathrm{N}_{2}$ até $110{ }^{\circ} \mathrm{C}$ e então a mistura redutora é admitida durante o aquecimento, até a temperatura final de redução (180, 190, 200 ou $\left.220^{\circ} \mathrm{C}\right)^{23}$. Neste caso, ao se atingir o patamar da temperatura de redução, já se tem uma conversão $\mathrm{a}_{0}$. É importante observar que este procedimento contém uma etapa de redução não isotérmica a partir de $110{ }^{\circ} \mathrm{C}$ até que seja atingido o patamar isotérmico de redução, a duração da etapa isotérmica depende da quantidade de material redutível remanescente da etapa não isotérmica. Procedimento 2: o gás redutor é admitido somente na temperatura de redução9 ${ }^{9}$ A amostra é aquecida em $\mathrm{N}_{2}$ até a temperatura de redução $\left(190,200\right.$ ou $\left.210{ }^{\circ} \mathrm{C}\right)$ quando a mistura redutora é admitida.

Neste estudo, foram utilizados os dois procedimentos com o objetivo de comparar os resultados. O aquecimento até o patamar isotérmico foi realizado a uma taxa de controlada de $10{ }^{\circ} \mathrm{C} \mathrm{min}^{-1}$ sob fluxo constante $\left(\mathrm{F}=20 \mathrm{~cm}^{3} \mathrm{~min}^{-1}\right)$ de uma mistura de $\mathrm{H}_{2} / \mathrm{N}_{2}$ de concentração $3 \%$ v/v. As curvas de redução foram obtidas representando-se graficamente a fração de $\mathrm{CuO}$ reduzida em função do tempo, se tomando por base a reação de redução do óxido de cobre a cobre metálico.

Os estudos em condições não-isotérmicas (Procedimento 3) foram realizados em condições similares ao isotérmico (aquecimento a uma taxa de $10{ }^{\circ} \mathrm{C} \mathrm{min}^{-1}$ sob uma mistura de $\mathrm{H}_{2} / \mathrm{N}_{2}$ de concentração $3 \% \mathrm{v} / \mathrm{v}$ ), mas naturalmente, sem nenhum patamar final de temperatura e com a mistura redutora sendo admitida a $110{ }^{\circ} \mathrm{C}$. Abaixo desta temperatura não ocorre redução, de forma que torna-se mais econômico e seguro utilizar o $\mathrm{N}_{2}$.

$\mathrm{O}$ catalisador $\mathrm{CuO} / \mathrm{ZnO} / \mathrm{Al}_{2} \mathrm{O}_{3}$, com diâmetro de partícula médio igual a $0,007 \mathrm{~cm}$ (estimado por peneiramento), e razão atômica $\mathrm{Cu} / \mathrm{Zn} / \mathrm{Al}=40 / 45 / 15$ altamente ativo para a reação de shift foi utilizado neste estudo. Este catalisador foi preparado pela Oxiteno S.A., segundo o método apresentado em Lima et al. ${ }^{8}$; a caracterização deste catalisador é descrita em Lima et al. ${ }^{8}$. Resumidamente, a etapa de coprecipitação foi realizada adicionando-se uma solução dos nitratos metálicos $\left(2,7 \mathrm{~mol} \mathrm{~L}^{-1}\right)$ a uma solução de carbonato de sódio (14\% em peso). O precursor assim obtido foi maturado por $6 \mathrm{~h}$ em $\mathrm{pH} 5$, lavado exaustivamente, tratado em estufa a $160^{\circ} \mathrm{C}$ por $4 \mathrm{~h}$ para remoção de água e calcinado por $3 \mathrm{~h}$ a $350{ }^{\circ} \mathrm{C}$. A velocidade de adição da solução dos nitratos foi de aproximadamente $1 \mathrm{~mL} \mathrm{~min}$. $^{-1}$.

$\mathrm{O}$ perfil de redução do catalisador $\mathrm{CuO} / \mathrm{ZnO} / \mathrm{Al}_{2} \mathrm{O}_{3}$ com razão atômica $\mathrm{Cu} / \mathrm{Zn} / \mathrm{Al}=40 / 45 / 15$ preparado por este procedimento apresenta, principalmente, a redução de duas espécies de $\mathrm{Cu}^{2+}$. Uma, o óxido de cobre livre ou "bulk" e a outra uma espécie de $\mathrm{Cu}^{2+}$ estabilizado pela alumina, identificados através do valor da energia de ativação do processo de redução. Estas espécies apresentam picos de redução a 200 e $231^{\circ} \mathrm{C}$. Sobreposta à redução da segunda espécie há uma terceira espécie $247^{\circ} \mathrm{C}$ não identificada, mas que pode ser atribuída ao início da redução gradual dos hidroxicarbonatos residuais, que em atmosferas redutoras decompõem-se a temperaturas abaixo de $623 \mathrm{~K}$. Comparando-se o perfil do sistema $\mathrm{CuO} /$ $\mathrm{ZnO} / \mathrm{Al}_{2} \mathrm{O}_{3}$ com sistemas modelo $\mathrm{CuO} / \mathrm{ZnO}$ e $\mathrm{CuO} / \mathrm{Al}_{2} \mathrm{O}_{3}$ preparados por um procedimento similar observa-se que a espécie cuja redução se inicia a $150{ }^{\circ} \mathrm{C}$ está presente em todos os três catalisadores, logo pode-se afirmar que se trata de uma espécie relacionada ao cobre livre. Quanto à influência na redução, tanto o zinco quanto o alumínio aceleraram a redução do $\mathrm{CuO}$ livre, mas no catalisador contendo alumina $\left(\mathrm{CuO} / \mathrm{Al}_{2} \mathrm{O}_{3}\right)$ um segundo pico de redução é observado. O perfil de redução (obtido nas mesmas condições dos perfis abaixo) do óxido de cobre puro, preparado pelo mesmo método utilizado para preparar as outras amostras, apresentou um pico de redução referente ao $\mathrm{CuO}$ a $229^{\circ} \mathrm{C}^{14,24}$.

\section{RESULTADOS E DISCUSSÃO}

\section{Estudo isotérmico}

A Figura 2 mostra as curvas de redução em função do tempo obtidas isotermicamente a diferentes temperaturas, admitindo-se a mistura de gás redutor $\left(\mathrm{H}_{2} / \mathrm{N}_{2}\right)$ durante a etapa de aquecimento (Procedimento 1). Ao atingir a temperatura desejada para o estudo da reação, este procedimento resulta em um catalisador parcialmente reduzido.
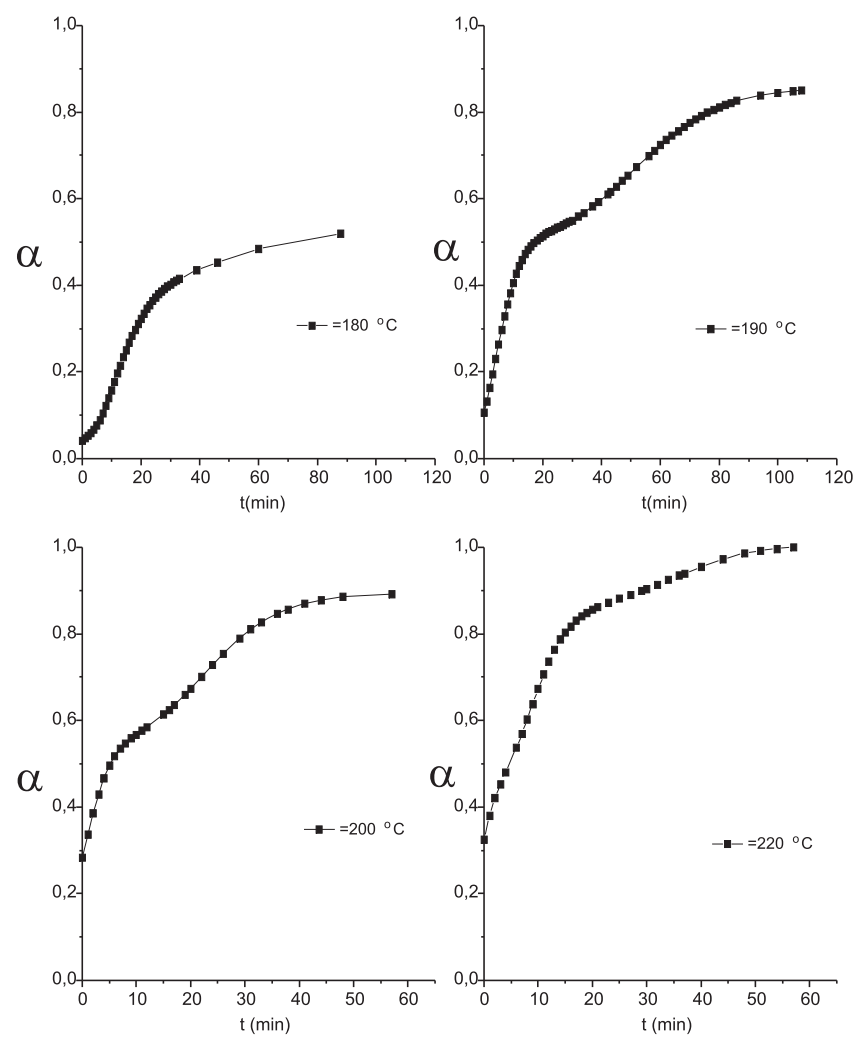

Figura 2. Reduções isotérmicas do catalisador $\mathrm{CuO} / \mathrm{ZnO} / \mathrm{Al}_{2} \mathrm{O}_{3}$ à diferentes temperaturas $(\alpha=$ grau de redução)

A $180{ }^{\circ} \mathrm{C}$ a curva de redução (Figura 2) apresenta a forma sigmoidal típica para reações topoquímicas ${ }^{10}$ ou autocatalíticas ${ }^{9}$, especialmente para a redução do $\mathrm{CuO}$; os três estágios do processo podem ser visualizados: Indução (de 0 a \pm 7 min): durante este período a taxa de reação é muito baixa. Como conseqüências da reação entre o hidrogênio e moléculas mais reativas de óxido de cobre (sítios de adsorção ${ }^{18}$ ), os átomos de cobre localizam-se preferencialmente nos cristais de óxido de cobre. Quando a concentração de átomos de cobre reduzidos $\left(\mathrm{Cu}^{\circ}\right)$ atinge um valor crítico, eles associam-se e núcleos de cobre são formados por cristalização, desenvolvendo a interface $\mathrm{Cu} / \mathrm{CuO}$. Allen et al. ${ }^{25}$ consideraram que este período representa uma reação superficial e postularam que a difusão de vacâncias aniônicas da superfície para o interior do sólido pode ser a etapa limitante deste período. Aceleração (de \pm 7 a \pm 
$30 \mathrm{~min})$ : este é o período imediatamente posterior ao de indução, no qual a interface $\mathrm{Cu} / \mathrm{CuO}$ se desenvolve. Nele há um significativo aumento na taxa de reação provocado pelo crescimento dos núcleos metálicos formados durante o período de indução ${ }^{16}$. Neste período poucos núcleos são formados e o consumo de hidrogênio observado é devido ao crescimento de núcleos pré-existentes, o crescimento dos núcleos tem uma taxa de consumo de hidrogênio muito maior que a formação. Atenuação (a partir de $\pm 30 \mathrm{~min}$ ): é a última etapa apresentada na isoterma de redução, caracterizada por um drástico decréscimo na taxa de reação a baixas temperaturas e por, praticamente, uma parada abrupta na taxa de reação a altas temperaturas ${ }^{9}$. Este período está relacionado a importantes transformações físicas do sistema, como recristalização e aglomeração ${ }^{10}$.

A $180{ }^{\circ} \mathrm{C}$ apenas a primeira espécie de cobre, atribuído ao $\mathrm{CuO}$ livre ${ }^{8}$, é reduzida. A partir de $190{ }^{\circ} \mathrm{C}$, a redução ocorre em dois estágios, caracterizados por diferentes coeficientes angulares das etapas de aceleração. No primeiro estágio a espécie $\mathrm{CuO}$ livre é reduzida, e no segundo estágio uma espécie de $\mathrm{Cu}^{2+}$, identificada como hipoteticamente $\mathrm{CuAl}_{2} \mathrm{O}_{4}{ }^{12}$ é lentamente reduzida. A partir desta temperatura perdeu-se totalmente a informação relativa ao período de indução, pois a conversão iniciada na etapa de redução do $\mathrm{CuO}$ anterior confunde-se com a redução desta segunda espécie. A redução de diferentes espécies de $\mathrm{Cu}^{2+}$ pode ser visualizada também através do gráfico da derivada da conversão em função do tempo (Figura 3).
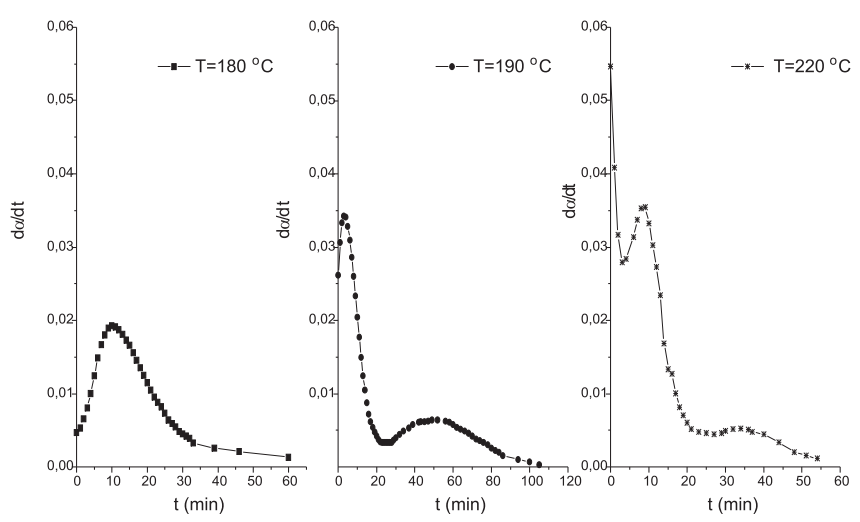

Figura 3. Derivada da conversão em função do tempo

Na segunda estratégia experimental, o catalisador é aquecido em gás não-redutor $\left(\mathrm{N}_{2}\right)$ até que a temperatura da reação é atingida, só então o gás de reação é admitido (Procedimento 2). Estas curvas também apresentaram a forma sigmoidal (Figura 4) característica para reações topoquímicas, porém, conforme se constata pelas inclinações das curvas de grau de redução em função do tempo (Figuras 2 e 4), a taxa de reação observada foi bem mais alta do que quando o gás foi admitido durante o aquecimento. Quando o gás é admitido na temperatura da reação a quantidade de núcleos formados é bem maior, acelerando a redução.

Inicialmente, estudou-se o processo de redução para o gás admitido durante o aquecimento. $\mathrm{O}$ resultado obtido a $220{ }^{\circ} \mathrm{C}$ não foi utilizado, pois ao atingir esta temperatura a redução da primeira espécie de $\mathrm{Cu}^{2+}$ já estava em período desaceleratório, não sendo possível calcular a velocidade empírica.

Utilizando o método da constante empírica da taxa de reação $0^{21}$, a energia de ativação obtida por regressão linear foi de $71 \mathrm{~kJ} \mathrm{~mol}^{-1}$ para a redução do óxido de cobre. Para a segunda espécie redutível presente no catalisador, a energia de ativação calculada foi de 102 $\mathrm{kJ} \mathrm{mol}^{-1}$, significativamente maior que a energia de ativação do $\mathrm{CuO}$ livre, confirmando a estabilização de parte dos íons $\mathrm{Cu}^{2+}$ pre-

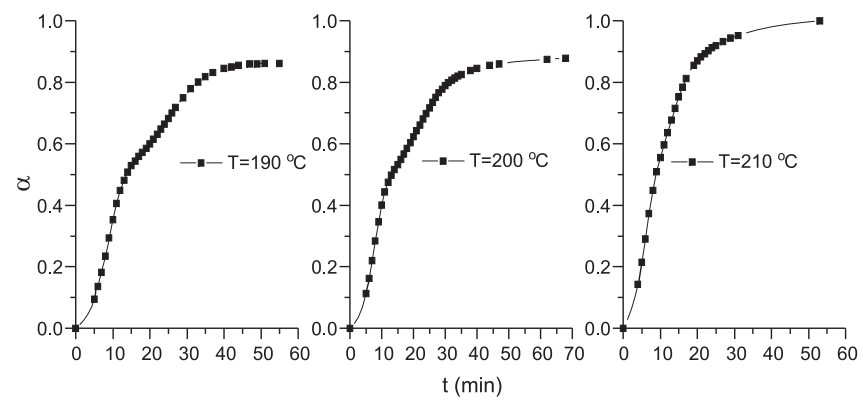

Figura 4. Reduções isotérmicas a diferentes temperaturas $(\alpha=$ grau de redução)

sentes no catalisador, possivelmente por íons aluminato oriundos da espécie hipotética $\mathrm{CuAl}_{2} \mathrm{O}_{4}$.

A escolha de um modelo mecanístico dentro de um conjunto de modelos passíveis de descrever o sistema é uma parte específica da estatística aplicada à experimentação, conhecida como discriminação de modelos, que conta com métodos próprios para seleção do modelo mais apropriado. Neste trabalho optou-se pela utilização da abordagem tradicional, em virtude da magnitude do nosso erro experimental na determinação da cinética isotérmica.

Serão mostradas detalhadamente as escolhas dos modelos para as reduções a 180 e $190{ }^{\circ} \mathrm{C}$, com o hidrogênio adicionado durante o aquecimento (Procedimento 1). Nos outros casos do mesmo procedimento isotérmico, a 200 e $220{ }^{\circ} \mathrm{C}$, serão apresentados apenas os resultados finais. Por motivos operacionais, o primeiro estágio da redução (indução) não será analisado, pois, em função da rapidez desta etapa, não foi possível obter pontos experimentais suficientes nos primeiros instantes da reação.

A Figura 5 apresenta a curva de redução a $180{ }^{\circ} \mathrm{C}$. O segundo estágio (aceleração) foi analisado utilizando as equações descritas anteriormente para as duas espécies de $\mathrm{Cu}^{+2}$. São apresentados a seguir alguns resultados da regressão (o coeficiente angular - constante da taxa - e o somatório dos quadrados dos resíduos - $\mathrm{SS}_{\text {res }}$ ) para cada uma destas equações e para as duas espécies de cobre redutíveis (Tabelas 2 e 3 ).

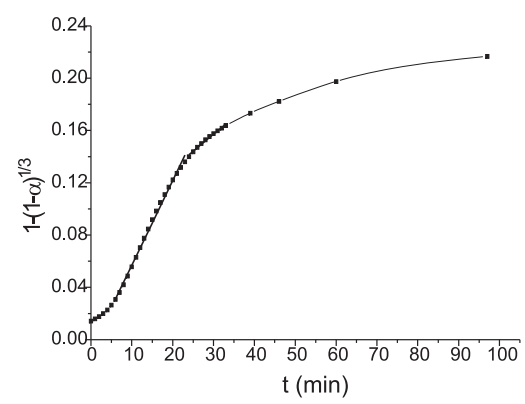

Figura 5. Comparação entre o modelo escolhido e os dados experimentais

Tabela 2. Resultados da discriminação de modelos para a redução isotérmica do óxido de cobre

\begin{tabular}{lcc}
\hline$g_{(\alpha, \mathbf{d})}=k_{(\mathrm{T}, \mathbf{b})} \mathrm{t}$ & $k_{(\mathrm{T}, \mathbf{b})}$ & $\mathrm{SS}_{\mathrm{res}}$ \\
\hline$\alpha$ & 0,01628 & 0,00098 \\
{$[-\ln (1-\alpha)]^{1 / 2}$} & 0,02135 & 0,00375 \\
{$[-\ln (1-\alpha)]^{1 / 3}$} & 0,01810 & 0,00416 \\
{$[-\ln (1-\alpha)]^{1 / 4}$} & 0,01532 & 0,00361 \\
$1-(1-\alpha)^{1 / 2}$ & 0,00929 & 0,00019 \\
$1-(1-\alpha)^{1 / 3}$ & 0,00647 & 0,00008 \\
$\ln [\alpha /(1-\alpha)]+\mathrm{C}$ & 0,09981 & 0,20501 \\
\hline
\end{tabular}


Tabela 3. Resultados da discriminação de modelos para a redução isotérmica do aluminato de cobre

\begin{tabular}{lcc}
\hline$g_{(\alpha, \mathbf{d})}=k_{(\mathrm{T}, \mathbf{b})} \mathrm{t}$ & $k_{(\mathrm{T}, \mathrm{b})}$ & $\mathrm{SS}_{\text {res }}$ \\
\hline$\alpha$ & 0,00398 & 0,00033 \\
{$[-\ln (1-\alpha)]^{1 / 2}$} & 0,00464 & 0,00045 \\
{$[-\ln (1-\alpha)]^{1 / 3}$} & 0,00344 & 0,00026 \\
{$[-\ln (1-\alpha)]^{1 / 4}$} & 0,00272 & 0,00017 \\
$1-(1-\alpha)^{1 / 2}$ & 0,00259 & 0,00012 \\
$1-(1-\alpha)^{1 / 3}$ & 0,00189 & 0,00006 \\
$\ln [\alpha /(1-\alpha)]+\mathrm{C}$ & 0,01646 & 0,00627 \\
\hline
\end{tabular}

As equações que melhor descreveram os dados experimentais foram as correspondentes aos modelos de crescimento interfacial, especialmente a Equação 2 (modelo de crescimento isotrópico), pois apresentou para as duas espécies de $\mathrm{Cu}^{2+}$ o menor somatório do quadrado dos resíduos. $\mathrm{O}$ ajuste deste modelo aos dados experimentais pode ser avaliado na Figura 5. A parte da curva não descrita pelo modelo está relacionada à etapa da reação com significativa influência de transformações físicas do sistema, como recristalização e aglomeração, de elevada complexidade e que não foram tratadas neste trabalho.

Este mesmo procedimento foi repetido para a curva obtida a $190{ }^{\circ} \mathrm{C}$, com o gás admitido durante o aquecimento. Para a redução da espécie atribuída ao óxido de cobre, o modelo isotrópico de crescimento do cristal (Equação 2) também descreveu satisfatoriamente os dados experimentais. Os mesmos modelos foram testados para a espécie sugerida como $\mathrm{Cu}^{2+}$ em forte interação com a alumina. Novamente a equação obtida do modelo isotrópico de crescimento do cristal foi a que resultou em menor soma dos quadrados dos resíduos, indicando uma boa aderência dos pontos experimentais ao modelo. A equação de Avrami com $n=4$ e a equação baseada no crescimento bidimensional do cristal (Equação 4) também descreveram satisfatoriamente os resultados experimentais. Escolheu-se o modelo isotrópico para descrever estes dados, pois foi o que apresentou menor soma dos quadrados dos resíduos $\left(\mathrm{SS}_{\text {res }}\right)$ e já foi utilizado com sucesso, na literatura, para descrever a redução desta mesma espécie ${ }^{26}$.

$\mathrm{O}$ ajuste do modelo aos dados experimentais pode ser avaliado na Figura 6.

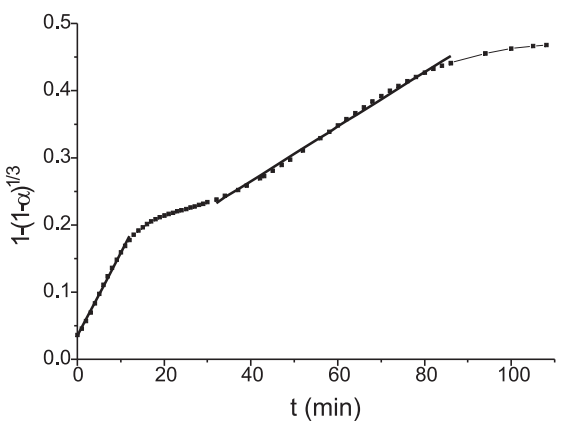

Figura 6. Comparação entre o modelo escolhido e os dados experimentais

As energias de ativação obtidas utilizando o modelo do crescimento isotrópico do cristal foram $65 \mathrm{~kJ} \mathrm{~mol}^{-1}$ para a espécie identificada como $\mathrm{CuO}$ livre e $92 \mathrm{~kJ} \mathrm{~mol}^{-1}$ para a espécie $\mathrm{CuAl}_{2} \mathrm{O}_{4}$. Estes valores de energia de ativação estão em boa concordância com a faixa de valores obtidos na literatura para a redução destas duas espécies ${ }^{12,26,27}$ e com os valores obtidos por redução termoprogramada.

\section{Estudo não-isotérmico}

A Figura 7 apresenta um perfil de redução termoprogramada típico para este catalisador (Procedimento 3). Pode-se observar o início do consumo de hidrogênio a, aproximadamente, $100{ }^{\circ} \mathrm{C}$ (373 K) possivelmente devido à decomposição de espécies superficiais de óxido de cobre finamente dispersas ou com reducibilidade promovidas pelo óxido de zinco ${ }^{13}$; a seguir, a redução das duas espécies de $\mathrm{Cu}^{2+}$, temperaturas de máxima redução em $200{ }^{\circ} \mathrm{C}(473 \mathrm{~K})$ e $230{ }^{\circ} \mathrm{C}(503 \mathrm{~K})$, respectivamente; e um desvio da linha de base até o final da análise, atribuído à decomposição de carbonatos residuais, como observado anteriormente em um sistema similar ${ }^{28}$.

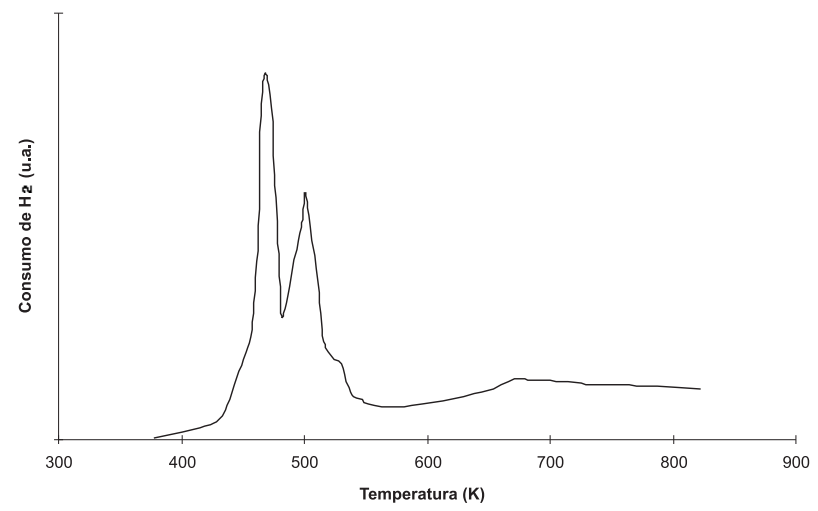

Figura 7. Perfil de redução típico da amostra $\mathrm{CuO} / \mathrm{ZnO} / \mathrm{Al}_{2} \mathrm{O}_{3}(\mathrm{Cu} / \mathrm{Zn} / \mathrm{Al}=$ $40 / 45 / 15, \beta=4{ }^{\circ} \mathrm{C} \mathrm{min}^{-1}, F / W=0,3 \mathrm{Lg}^{-1} \mathrm{~min}^{-1}$ )

Se a temperatura do reator aumentar linearmente com o tempo e a equação da taxa for escrita na forma padrão de Arrhenius, no ponto onde a taxa de redução é máxima tem-se ${ }^{22}$ :

$\ln \frac{T_{m}^{2} C_{m}^{p}}{\beta}+\ln q S_{m}^{q-1}=\frac{E}{R T_{m}}+\ln \frac{E}{R A}$

onde o subscrito $m$ refere-se ao ponto de máxima taxa de reação; $E$ é a energia de ativação da reação de redução; $T$ é a temperatura em Kelvin; $A$ é o fator pré-exponencial; $p$ a ordem de reação em relação ao hidrogênio; $q$ a ordem de reação em relação ao sólido redutível; $R$ a constante universal dos gases; $\beta$ a taxa de aquecimento; $\mathrm{Cm}$ a concentração de hidrogênio no ponto de máxima taxa de reação.

Conhecendo-se os valores de $p$ e $q$, um gráfico linear do lado esquerdo da equação versus $1 / T_{m}$ terá como coeficiente angular $E$ / $R$ e coeficiente linear $\ln (E / R A)$. Desta forma, pode-se obter os valores numéricos da energia de ativação $(E)$ e do fator pré-exponencial (A).

Normalmente $p$ e $q$ não são conhecidos, admitiu-se inicialmente que a ordem de reação em relação ao hidrogênio e ao sólido são unitárias, ou seja, $q=1$ e $p=1$, obtendo-se a equação:

$\ln \frac{T_{m}^{2} C_{m}}{\beta}=\frac{E}{R T_{m}}+\ln \frac{E}{R A}$

A análise cinética só foi realizada para as duas principais espécies de $\mathrm{Cu}^{2+}$ presentes no catalisador, representadas por dois picos bem definidos.

Os experimentos realizados para estimação dos parâmetros cinéticos segundo a Equação 7 são apresentados na Tabela 4, onde $\beta$ é a taxa de aquecimento $\left(\mathrm{K} \mathrm{s}^{-1}\right) ; \mathrm{C}_{\mathrm{m} 1}$ e $\mathrm{C}_{\mathrm{m} 2}$ são as concentrações de hidrogênio nos pontos de máxima redução $\left(\mu \mathrm{mol} \mathrm{cm}{ }^{-3}\right) ; \mathrm{T}_{\mathrm{m} 1} \mathrm{e} \mathrm{T}_{\mathrm{m} 2}$ 
são as temperaturas nos pontos de máxima redução $\left({ }^{\circ} \mathrm{C}\right) . \mathrm{O}$ gráfico representando a regressão linear dos dados obtidos é apresentado na Figura 8 e os parâmetros cinéticos estimados são mostados na Tabela 5.

Tabela 4. Experimentos realizados para estimação dos parâmetros cinéticos

\begin{tabular}{lccccc}
\hline Experimento & $\begin{array}{c}\beta \\
\left(\mathrm{K} \mathrm{s}^{-1}\right)\end{array}$ & $\begin{array}{c}\mathrm{C}_{\mathrm{m} 1} \\
\left(\mu \mathrm{mol} \mathrm{cm}^{-3}\right)\end{array}$ & $\begin{array}{c}\mathrm{C}_{\mathrm{m} 2} \\
\left(\mu \mathrm{mol} \mathrm{cm}^{-3}\right)\end{array}$ & $\begin{array}{c}\mathrm{T}_{\mathrm{m} 1} \\
\left({ }^{\circ} \mathrm{C}\right)\end{array}$ & $\begin{array}{c}\mathrm{T}_{\mathrm{m} 2} \\
\left({ }^{\circ} \mathrm{C}\right)\end{array}$ \\
\hline 2 & 0,06 & 1,78 & 1,89 & 193 & 228 \\
8 & 0,06 & 1,77 & 1,88 & 189 & 226 \\
1 & 0,09 & 1,73 & 1,80 & 202 & 238 \\
10 & 0,09 & 1,69 & 1,77 & 199 & 238 \\
4 & 0,12 & 1,60 & 1,69 & 209 & 245 \\
9 & 0,12 & 1,48 & 1,67 & 211 & 245 \\
7 & 0,14 & 1,47 & 1,56 & 221 & 253 \\
3 & 0,14 & 1,59 & 1,61 & 217 & 251 \\
6 & 0,17 & 1,41 & 1,43 & 226 & 258 \\
5 & 0,17 & 1,43 & 1,52 & 224 & 256 \\
\hline
\end{tabular}

Tabela 5. Parâmetros cinéticos estimados

\begin{tabular}{lcc}
\hline Parâmetro cinético & $\begin{array}{c}\text { Primeira espécie } \\
\text { de } \mathrm{Cu}^{2+}\end{array}$ & $\begin{array}{c}\text { Segunda espécie } \\
\text { de } \mathrm{Cu}^{2+}\end{array}$ \\
\hline Energia de ativação (E) & $61 \pm 9 \mathrm{~kJ} \mathrm{~mol}^{-1}$ & $86 \pm 5 \mathrm{~kJ} \mathrm{~mol}^{-1}$ \\
Fator pré-exponencial (A) & $10^{7} 1 \mathrm{~mol}^{-1} \mathrm{~s}^{-1}$ & $10^{9} 1 \mathrm{~mol}^{-1} \mathrm{~s}^{-1}$ \\
\hline
\end{tabular}

* Com $95 \%$ de grau de confiança.
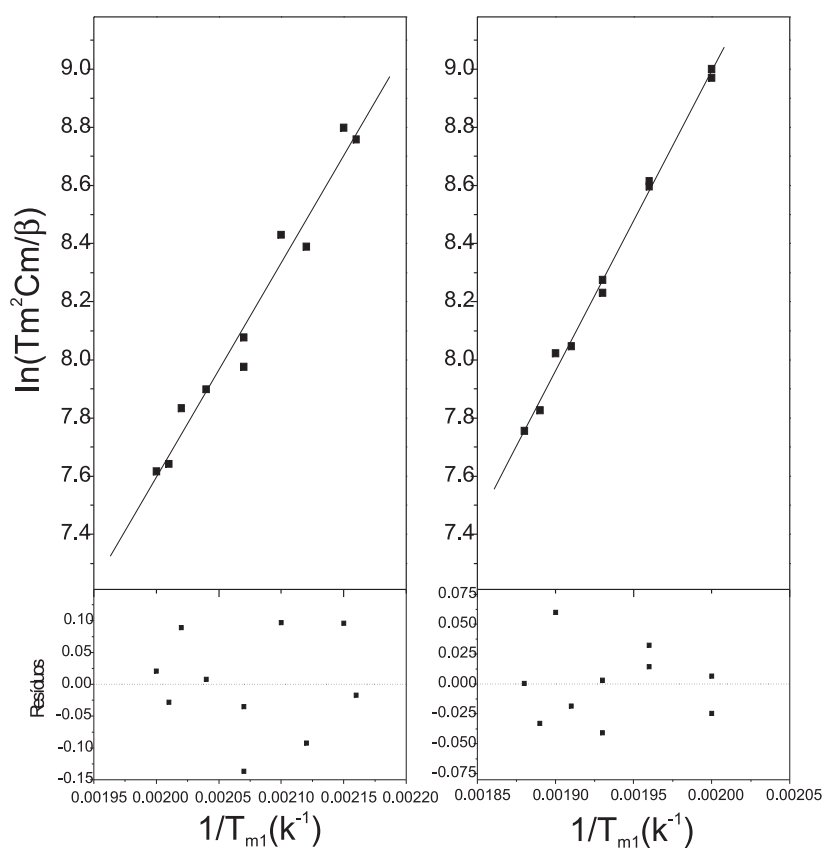

Figura 8. Curva de obtenção da energia de ativação pelo método da variação da taxa de aquecimento

A análise dos gráficos dos resíduos demonstra que não há qualquer tendência aparente nos resíduos. Este resultado indica que não há nenhuma violação da hipótese de normalidade do erro experimental e, por isso, o modelo descreve satisfatoriamente os dados experimentais.

Comparando-se os dados obtidos com os da literatura, pode-se afirmar que o primeiro pico de redução $\left(\mathrm{E}=61 \pm 9 \mathrm{~kJ} \mathrm{~mol}^{-1}\right)$ corresponde à redução do $\mathrm{CuO}$ livre ("bulk"), cuja energia de ativação varia na literatura de 40 a $80 \mathrm{~kJ} \mathrm{~mol}^{-1}$. A energia de ativação da redução da segunda espécie de $\mathrm{Cu}^{2+}\left(\mathrm{E}=86 \pm 5 \mathrm{~kJ} \mathrm{~mol}^{-1}\right)$ corresponde à energia de ativação da redução de íons cobre estáveis. Bueno et $a l .^{12}$ atribuem esta estabilização à formação de uma espécie hipotética $\mathrm{CuAl}_{2} \mathrm{O}_{4}$, na qual o $\mathrm{Cu}^{2+}$ está em forte interação com o íon aluminato.

Para verificar a hipótese da taxa de redução ser de primeira ordem em relação ao hidrogênio, realizaram-se experimentos adicionais com uma corrente redutora $\mathrm{H}_{2} / \mathrm{N}_{2}$ a $5 \%$ de $\mathrm{H}_{2}$ (v/v) (Procedimento 3). A equação utilizada para determinar a energia de ativação global do processo de redução do óxido de cobre mássico, representada por uma reta, foi capaz de descrever o efeito da variação da concentração de hidrogênio. Na redução do $\mathrm{Cu}^{2+}$ estabilizado, a equação utilizada também é capaz de descrever o efeito da variação da concentração de hidrogênio na corrente de alimentação na faixa estudada, indicando que a reação é de primeira ordem em relação ao hidrogênio para as duas espécies de $\mathrm{Cu}^{+2}$.

Comparando-se estes resultados com a literatura ${ }^{12}$ observa-se que ordem de reação um, em relação ao hidrogênio, na redução do óxido de cobre livre (ou puro) já foi observado. Por outro lado, até então, nenhum trabalho determinou a ordem de reação da redução do $\mathrm{Cu}^{2+}$ estabilizado.

\section{CONCLUSÕES}

Este trabalho apresentou a utilização do método de redução e isotérmica programada para obter, a partir de parâmetros cinéticos, informações relevantes do catalisador $\mathrm{CuO} / \mathrm{ZnO} / \mathrm{Al}_{2} \mathrm{O}_{3}$. Demonstrouse que este sistema é mais bem descrito pela equação derivada do modelo do crescimento interfacial isotrópico. Foram relacionadas duas espécies principais de $\mathrm{Cu}^{2+}$, uma de mais fácil redução identificada como óxido de cobre livre e outra de redução mais lenta, sugerindo uma estabilização do $\mathrm{Cu}^{2+}$ por uma outra espécie, sugerida como sendo a alumina. Os resultados de redução isotérmica demonstraram que a cinética de redução é melhor descrita pelo modelo do crescimento isotrópico do cristal para as duas espécies de $\mathrm{Cu}^{2+}$.

As energias de ativação calculadas para as reduções termoprogramadas e reduções isotérmicas apresentaram ótimas concordâncias para as duas espécies de óxido de cobre presentes no catalisador (aproximadamente 60 e $90 \mathrm{~kJ} \mathrm{~mol}^{-1}$ ). Foi evidenciado que a ordem de reação em relação ao hidrogênio é unitária.

\section{AGRADECIMENTOS}

M. Nele agradece ao CNPq pela bolsa de pesquisa e E. L. Moreno ao suporte através da bolsa do $\mathrm{CNPq} / \mathrm{RHAE}$.

\section{REFERÊNCIAS}

1. Chinchen, G. C.; Davies, L.; Oldman, R. J.; Andrews S. J.; Proceedings of the Sixth International Symposium on the Scientific Bases for the Preparation of Heterogeneous Catalysts, Louvain la Neuve: Belgium, 1994, vol. 1, p. 49.

2. Silva, A. M. T.; Castelo-Branco, I. M.; Quinta-Ferreira R. M.; Levec, J.; Chem. Eng. Sci. 2003, 58, 963.

3. Fujita, S.; Satrujo, A. M.; Shen, G. C.; Takezawa, N.; Catal. Lett. 1995, $34,85$.

4. Gineâs, M. J. L.; Amadeo, N.; Laborde, M.; Apesteguía, C. R.; Appl. Catal. 1995, 131, 283.

5. Andrew, S. P. S.; Chem. Eng. Sci. 1981, 36, 1431.

6. Klier, K.; Catal. Today 1992, 15, 361.

7. Campbell, J. S.; Craven, P.; Young, P. W.; Catalyst Handbook, Wolfe Scientific Books: London, 1970, p. 97.

8. Lima, A. G.; Nele, M.; Moreno, E. L.; Andrade, H. M. C.; App. Catal. 1998, 
$171,31$.

9. Naidu, S. R.; Technology 1972, 9, 123.

10. Naumov, Y. B.; Pavlov, Y. L.; Vasilevich, A. A.; Alekseev, A. M.; Novikov, E. A.; Shchibrya, G. G.; Kinet. Catal. 1986, 27, 818.

11. Ruggeri, O.; Trifiró, F.; Vaccari, A.; J. Solid State Chem. 1982, 42, 120.

12. Bueno, J. M. C.; Gazzano, M.; Coelho, M. G.; Vaccari, A.; Appl. Catal. 1993, 103, 69.

13. Fierro, G.; Jacono, M. L.; Inversi, M.; Porta, P.; Cioci, F.; Lavecchia, R.; Appl. Catal. 1996, 137, 327.

14. Nele, M. ; Moreno, E. L. ; Andrade, H. M. C.; Quim. Nova 2006, 29, 641.

15. Militiký, J.; Sestak, J.; Thermochim. Acta 1992, 203, 31

16. Boldyrev, V. V.; Bullens, M.; Delmon, B.; The Control of the Reactivity of Solids, Studies in Surface Science and Catalysis 2, Elsevier Scientific Publishing Company: Amsterdam, 1979.
17. Dollimore, D.; Thermochim. Acta 1992, 203, 7 .

18. Bond, W. D.; J. Phys. Chem. 1962, 66, 1573.

19. Vogue, H. H.; J. Catal. 1962, 1, 171.

20. Sesták, J.; Berggren, G.; Thermochim. Acta 1971, 3, 1.

21. Delmon, B.; Introduction a la Cinétique Hétérogène, Technoprint: Paris, 1969.

22. Jones, A.; McNicol, B.; Temperature Programmed Reduction for Solid Materials Characterization, Marcel Decker Inc.: New York, 1986.

23. Plewa, J.; Skrzypek J.; Chem. Eng. Sci. 1989, 44, 2817.

24. Nele, M.; Dissertação de Mestrado, Universidade Federal da Bahia, Brasil, 1995.

25. Allen, J. A.; Scaife, D. E.; J, Phys. Chem. 1954, 58, 667.

26. Gusi, S.; Trifiró, F.; Vaccari, A.; React. Solids 1986, 2, 59.

27. Pouchot, M. T.; Verhoeven, W.; Delmon, B.; Bull. Soc. Chim. Fr. 1966, 911.

28. Matter, P. H.; Braden, U. D. J.; Ozkan, S.; J. Catal. 2004, 223, 340. 